\title{
Dual X-ray absorptiometry whole body composition of adipose tissue in rheumatoid arthritis
}

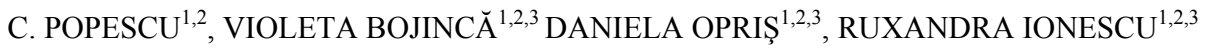 \\ 1"Sfânta Maria" Clinical Hospital, Bucharest \\ 2“"Carol Davila” University of Medicine and Pharmacy, Department of Internal Medicine and Rheumatology, Bucharest \\ ${ }^{3}$ Research Centre of the Pathology and Treatment of Systemic Rheumatic Diseases, Bucharest
}

\begin{abstract}
Aim. Rheumatoid arthritis (RA) may influence not only abdominal fat, but also whole body adiposity, since it is associated with chronic inflammation and disability. The study aims to evaluate the whole body adiposity of RA patients and to assess potential influences of disease specific measures.

Methods. The study was designed to include Caucasian postmenopausal female RA patients and age-matched postmenopausal female controls. Each subject underwent on the same day clinical examination, laboratory tests, whole body dual X-ray absorptiometry (DXA) composition and physical activity estimation using a self-administered questionnaire.

Results. A total of 107 RA women and 104 matched controls were included. Compared to controls, the RA group had less physical activity and a higher prevalence of normal weight obesity. Overfat RA women had a significantly higher toll of inflammation, disease activity, glucocorticoid treatment and sedentary behavior. RA women with inflammation, glucocorticoid treatment and higher disease activity class had higher whole body and trunk adipose tissue indices and higher prevalence of overfat status. Glucocorticoid treatment, inflammation, disease duration and severity correlated with whole body adipose tissue and significantly predicted high adiposity content and overfat phenotypes.

Conclusions. RA disease duration and severity are associated with higher whole body and regional adiposity. Low-dose glucocorticoid treatment seems to contribute to adiposity gain and redistribution. Clinicians may need to assess body composition and physical activity in RA patients in order to fully manage cardiovascular outcomes and quality of life.
\end{abstract}

Key words: rheumatoid arthritis, DXA body composition, adipose tissue, obesity.

\section{INTRODUCTION}

Rheumatoid arthritis (RA) is a progressive auto-immune disease characterized by chronic inflammation which leads to joint deformity, disability, distress and socio-economic costs. RA is also associated with modifications of the body composition. The mass and distribution of fat, muscle and bone are equally changed by RA [1], even in the early stages [2-4]. The RA-adipose tissue interaction entails central adiposity, the metabolic syndrome (MetS) and obesity classically defined by a body mass index (BMI) above $30 \mathrm{~kg} / \mathrm{m}^{2}$. It seems that BMI-defined obesity has a comparable prevalence among RA and general populations [5], a figure which may be underestimated by the fact that the classical BMI cutoffs are too high for RA patients (for a given body fat content, RA patients had a BMI with $2 \mathrm{~kg} / \mathrm{m}^{2}$ lower than controls, probably due to the loss of muscle mass and to the gain of fat mass) [6]. Another drawback of BMI is the lack of information regarding the actual amount and distribution of fat, knowing that central obesity and the MetS are highly prevalent in RA patients [7-9]. The study of these conditions is relevant clinically, since adipose tissue disturbances increase the cardiovascular risk [10], but also for fundamental research, since there are reports of bidirectional pathological relationships [11]. The assessment of body composition in RA was mostly done using anthropometry and bioimpedance [12, 13], while the use of dual X-ray absorptiometry (DXA) technique was limited [3, 4, 14, 15]. Most of the studies included both genders, despite the facts that RA is more prevalent among women and that body composition differs significantly between genders. In this context,we hypothesized that there must be a specific effect of RA measures on whole body adiposity, not exclusively on abdominal fat, since the disease is associated with a number of factors which can influence the adipose tissue (e.g. inflammation, disability, glucocorticoids). Therefore, the study aims to evaluate the whole body adiposity RA patients using DXA and to assess potential influences of disease measures on body composition phenotypes. 


\section{METHODS}

\subsection{PATIENTS AND CRITERIA}

The study was designed to include all the female patients admitted to our Rheumatology Department (Research Centre of the Pathology and Treatment of Systemic Rheumatic Diseases - RCRD, Bucharest) in the random order of presentation between May and August 2014. The inclusion criteria were: female sex (since RA is more frequent in women and since men have largely different body composition phenotypes than women), Caucasian race, postmenopausal status, 2010 ACR/EULAR classification criteria for RA [16], a RA disease duration longer than 6 months. Age-matched healthy Caucasian post-menopausal female subjects were randomly selected and invited to participate in the study using the records of general practitioners associated with RCRD from the same geographic area. The following exclusion criteria were applied for both groups: weight $>150 \mathrm{~kg}$ (DXA table weight limit); current cancer; digestive pathology (gastrectomy, bariatric or intestinal surgery, primary biliary cirrhosis, celiac disease, malabsorption); chronic obstructive lung disease; severe heart failure (New York Heart Association classifycation $\geq 3$ ); moderate-severe chronic kidney disease (glomerular filtration rate $<60 \mathrm{~mL} / \mathrm{min} / 1.73 \mathrm{~m}^{2}$ ); endocrine abnormalities (hyperthyroidism, hyperparathyroidism, hypogonadism); psychiatric eating disorder, therapy with any of the following drugs in the last 6 months: estrogen-replacement therapy, glucocorticoids exceeding $7.5 \mathrm{mg}$ /day prednisone equivalent, antipsychotics, orlistat, lorcaserin, exogenous insulin. Each patient gave written informed consent and the study was approved by the local ethics committee. The clinical examination, laboratory tests and DXA body composition analysis were done on the same day for each patient.

\subsection{CLINICAL EXAMINATION}

A clinical interview and a review of the medical history recorded age, smoking status, disease duration, duration of morning stiffness, patient global self assessment of general health and disease activity (visual analog scale), treatment regimes and extra-articular manifestations (rheumatoid nodules, vasculitis, neurologic, pulmonary, cardiac, renal or ophthalmologic involvement). Quality of life was assessed using a self-administered modified health assessment questionnaire (HAQ) [17]. A single senior author rheumatologist (DO) performed systematical clinical examinations, which focused on anthropometrics and RA variables. The classic anthropometric indices such as height, weight, waist and hip circumferences (WC, HC) were measured in upright anatomical position, light clothes, without shoes, using a mechanical scale $(0.1 \mathrm{~kg}$ maximal error $)$, a stadiometer $(0.3 \mathrm{~cm}$ maximal error) and a centimeter graded stretch resistant tape. For WC, the tape was placed horizontally and directly on skin, midway between the last rib and the iliac crest, and read at the end of a normal exhalation. For HC, the tape was placed horizontally around the widest portion of the buttocks. The waist-to-hip ratio (WHR) was calculated dividing $\mathrm{WC}$ to $\mathrm{HC}$. Body mass index (BMI) was calculated dividing weight by the square of height. Obesity and overweight were defined using the World Health Organization (WHO) cutoffs of BMI $\geq$ $30 \mathrm{~kg} / \mathrm{m}^{2}$ and $25 \mathrm{~kg} / \mathrm{m}^{2}$ respectively. The clinical examination accounted for the number of painful and swollen joints (bilateral proximal interphalangeal, metacarpophalangeal, wrist, elbow, shoulder, knee joints) and identified patients with RA joint deformities of their hands (henceforth designated "joint deformity"): fixed flexion contracture, ulnar deviation, "swan neck", "boutonniere", "Z" thumb, other luxations and subluxations, impaired range of motion, arthritis mutilans. A visual analog scale indication of the global disease activity was recorded by the evaluator. A single senior author rheumatologist (VB) identified radiological damage on standard postero-anterior X-ray images of the hands and feet. The Simple Erosion Narrowing Score (SENS) was calculated using the method proposed by van der Heijde et al. [18].

\subsection{LABORATORY MEASURES}

All the included patients underwent morning venipuncture and blood samples were tested with commercial kits for routine blood chemistry and complete blood count, erythrocyte sedimentation rate (ESR; Westergren method), C-reactive protein (CRP; nephelometric method), IgM rheumatoid factor $(\mathrm{RF})$ and anti-citrullinated peptide antibodies (ACPA; enzyme-linked immunosorbent assay). Inflammation was classified if either CRP or VSH were above the upper limit of normal $(5 \mathrm{mg} / \mathrm{L}$ and $30 \mathrm{~mm} / \mathrm{h}$ respectively), in the absence of other causes than RA. Using the clinical and laboratory measures, RA activity was assessed using two composite tools: disease activity score (DAS28; remission $\leq 2.6$; low disease activity - LDA 2.63.2; moderate disease activity - MDA 3.2-5.1; high 
disease activity - HDA > 5.1) [19], and clinical disease activity index (CDAI; remission $\leq 2.8$; LDA 2.9-10; MDA 10.1-22; HDA $\geq 22.1$ ) [20].

\subsection{DXA WHOLE BODY COMPOSITION}

Body composition was evaluated by whole body less head DXA with a Lexxos C05LX223 densitometer. All the scans were performed by a single certified clinical densitometrist (CP; 0.48\% variation coefficient). Daily calibration and quality control tests were performed according to the manufacturer's recommendations and different regions of interest were manually checked for maximal reliability [21]. The patients were required to wear light clothing, without metal or plastic, and were scanned in the morning, after nocturnal fast, micturition and 5-10 minutes of supine rest on the examination table, in the absence of radioactive or radiocontrast investigations in the last week. Data records included whole body and regional (trunk, pelvis, arms, legs) variables such as adipose tissue density/mass/area/percent (wbATD/M/A/P). Appendicular adipose mass (AAM) was calculated as the sum of the adipose tissue masses of all four members, while the appendicular adipose density (AAD) was calculated as the arithmetic mean of the adipose tissue densities of all four members. Fat mass index (FMI) was calculated in two ways: dividing wbATM to weight (FMI1) or to square height (FMI2). Fat distribution ratio (FDR) was calculated either by dividing trunk adipose mass (TAM) by AAM (FDR1) or by dividing pelvic ATM by AAM (FDR2). Using the concepts of Lorenzo et al. [22], the patients were further categorized with normal weight obesity (NWO; BMI $<25 \mathrm{~kg} / \mathrm{m}^{2}$ and wbATP $>30 \%$ ) and pre-obese obesity (POO; BMI $>25 \mathrm{~kg} / \mathrm{m}^{2}$ and wbATP $>30 \%$ ). The metabolic syndrome (MetS) was defined using the 2006 International Diabetes Foundation criteria [23]. Using the DXA-derived cutoffs of wbATP proposed by Gallagher et al. [24] and the bioelectrical impedance analysis (BIA) cutoffs of FMI2 and wbATP proposed by Schutz et al. [25] and by Abernathy et al. [26] respectively, over $95 \%$ of our RA patients were overfat. In the absence of specific Romanian population cutoffs for whole body composition, we used our non-RA group as reference population. Accordingly, overfat status was classified either with a percent cutoff (wbATP above the $66^{\text {th }}$ percentile of the reference group, corresponding to $65.71 \%$ ) or with an absolute cutoff (FMI2 above the $66^{\text {th }}$ percentile of the reference group, corresponding to $18.51 \mathrm{~kg} / \mathrm{m}^{2}$ ).

\subsection{PHYSICAL ACTIVITY ESTIMATION}

Physical activity was estimated using the self-administered Global Physical Activity Questionnaire version 2 (GPAQ2), developed by WHO, with a total of 16 questions in 4 activity sections (work, travel, recreational, sedentary) [27, 28]. The English questionnaire was translated into Romanian by the authors and back-translated by an independent authorized translator. The final version of the Romanian version was pre-tested on a random sample of 15 healthy employees and 15 RA patients from RCRD.

\subsection{STATISTICS}

Data distribution normality was assessed using descriptive statistics, normality, stem-andleaf plots and the Lillefors corrected KolmogorovSmirnov test. Qualitative data were expressed as "absolute value (percentage of group)" and were studied using cross-tabs with $\chi^{2}$ or Fisher tests. Non-normally distributed scale data were reported as "median (interquartile range)" and their correlations and differences were assessed using nonparametric tests: bivariate Spearman and partial correlations for two scale variables (in order to exclude the effect of confounders on adipose tissue indices, these partial correlations were done controlling for age, BMI, physical activity, smoking and glucocorticoid use); Mann-Whitney $\mathrm{U}$ and KruskalWallis for differences of scale variables in groups with two (e.g. smoking) or more categories (e.g. RA disease activity). To determine which categories of multi-level nominal variables produced significant $\chi^{2}$ or Mann-Whitney tests, one-way ANOVA was used with post-hoc analysis (Tukey and Bonferroni multiple comparisons). To assess the independent predictive capacity of RA-variables, logistic regression models were created using adipose tissue phenotypes (binary logistic regression) or adipose tissue scale variables (standard multivariate linear regression) as dependents and RA variables and confounding variables as covariates; the scale variables included in the models were normalized using arithmetic functions (indicated in text). All tests were considered significant if $p<0.05$ and were done using IBM SPSS v.20 (IBM Inc., Armonk, N.Y., 2010) for Windows and GraphPad Prism 6.0 (GraphPad Software, La Jolla California, USA). 


\section{RESULTS}

\subsection{GENERAL CHARACTERISTICS}

The study included 107 RA postmenopausal female patients and 104 matched controls with regard to age and postmenopausal status (Table 1). Compared to controls, the RA group had a lower median BMI, less physical activity and a higher prevalence of NWO. Even though taken independently whole body adipose tissue indices, waist and hip circumferences did not differ significantly; when the former are normed by the latter significant differences appear (for example when wbATM was divided by the square of $\mathrm{HC}$ ).

Table 1

General characteristics and comparison of the study groups

\begin{tabular}{|c|c|c|c|}
\hline & $n o n-R A(n=104)$ & $R A(n=107)$ & $p$ \\
\hline age (years) & $56(48-78)$ & $56(46-76)$ & 0.718 \\
\hline smoking (n) & $21(20.2 \%)$ & $18(16.8 \%)$ & 0.098 \\
\hline height (m) & $1.58(0.08)$ & $1.60(0.08)$ & 0.086 \\
\hline weight $(\mathrm{kg})$ & $72(23)$ & $69(7)$ & 0.108 \\
\hline BMI $\left(\mathrm{kg} / \mathrm{m}^{2}\right)$ & $28.6(7.1)$ & $26.7(6.3)$ & 0.007 \\
\hline BMI normal weight (n) & $17(16.3 \%)$ & $34(31.8 \%)$ & 0.028 \\
\hline BMI overweight (n) & $41(39.4 \%)$ & $48(44.8 \%)$ & 0.491 \\
\hline BMI obesity (n) & $46(44.2 \%)$ & $25(23.3 \%)$ & 0.007 \\
\hline WC (cm) & $95.5(18.5)$ & $93.5(14.9)$ & 0.494 \\
\hline $\mathrm{HC}(\mathrm{cm})$ & $106(16.3)$ & $106(13)$ & 0.739 \\
\hline WHR & $0.89(0.06)$ & $0.88(0.07)$ & 0.259 \\
\hline MetS (n) & $65(62.5 \%)$ & $63(58.9 \%)$ & 0.711 \\
\hline wbATD $\left(\mathrm{g} / \mathrm{cm}^{2}\right)$ & $7.86(2.76)$ & $7.61(2.22)$ & 0.276 \\
\hline wbATM (kg) & $40.3(20.2)$ & $39.5(15.2)$ & 0.330 \\
\hline wbATA $\left(m^{2}\right)$ & $0.52(0.07)$ & $0.52(0.06)$ & 0.454 \\
\hline wbATP (\%) & $61.7(13.1)$ & $62.7(13.9)$ & 0.758 \\
\hline $\operatorname{TAD}\left(\mathrm{g} / \mathrm{cm}^{2}\right)$ & $13.1(9.1)$ & $12.2(8.1)$ & 0.252 \\
\hline TAM $(\mathrm{kg})$ & $0.91(0.63)$ & $0.93(0.55)$ & 0.758 \\
\hline $\operatorname{ATD}_{\text {pelvis }}\left(\mathrm{g} / \mathrm{cm}^{2}\right)$ & $11.8(4.3)$ & $11.1(3.9)$ & 0.207 \\
\hline $\operatorname{ATM}_{\text {pelvis }}(\mathrm{kg})$ & $7.7(5.1)$ & $7.7(3.5)$ & 0.985 \\
\hline $\operatorname{AAD}\left(\mathrm{g} / \mathrm{cm}^{2}\right)$ & $5.75(1.41)$ & $5.64(1.31)$ & 0.509 \\
\hline AAM $(k g)$ & $19.7(6.1)$ & $19.2(6.8)$ & 0.258 \\
\hline FDR1 (\%) & $4.53(2.38)$ & $4.78(2.32)$ & 0.170 \\
\hline FDR2 (\%) & $39.4(13.5)$ & $40.4(10.2)$ & 0.175 \\
\hline NWO (n) & $17(16.3 \%)$ & $34(31.8 \%)$ & 0.028 \\
\hline POO (n) & $41(39.4 \%)$ & $46(42.9 \%)$ & 0.599 \\
\hline FMI1 (\%) & $57.3(11.9)$ & $58.1(13.2)$ & 0.904 \\
\hline FMI2 $\left(\mathrm{kg} / \mathrm{m}^{2}\right)$ & $16.7(6.9)$ & $15.5(5.9)$ & 0.123 \\
\hline FMI2-overfat (n) & $35(33.7 \%)$ & $26(24.3 \%)$ & 0.171 \\
\hline wbATP-overfat (n) & $35(33.7 \%)$ & $37(34.6 \%)$ & 0.956 \\
\hline $\mathrm{ATD}_{\text {pelvis }} / \mathrm{WC}\left(\mathrm{mg} / \mathrm{cm}^{3}\right)$ & $12.6(2.9)$ & $11.9(3.1)$ & 0.040 \\
\hline $\mathrm{wbATM} / \mathrm{HC}^{2}\left(\mathrm{~kg} / \mathrm{m}^{2}\right)$ & $3.64(0.77)$ & $3.49(0.69)$ & 0.045 \\
\hline $\mathrm{ATD}_{\text {pelvis }} / \mathrm{HC}\left(\mathrm{mg} / \mathrm{cm}^{3}\right)$ & $108(30)$ & $105(29)$ & 0.043 \\
\hline $\mathrm{AAM} / \mathrm{HC}^{2}\left(\mathrm{~kg} / \mathrm{m}^{2}\right)$ & $1.73(0.36)$ & $1.67(0.25)$ & 0.032 \\
\hline TPA (kMET-min/week) & $5.2(0.7-13.7)$ & $4.7(0.5-11.3)$ & 0.041 \\
\hline MTT (min/week) & $14(0-154)$ & $11(0-103)$ & 0.045 \\
\hline SB (min/day) & $120(60-480)$ & $300(30-660)$ & 0.033 \\
\hline \multicolumn{4}{|l|}{ levels of physical activity } \\
\hline low (n) & $7(6.7 \%)$ & $14(13.1 \%)$ & \\
\hline moderate (n) & $34(32.7 \%)$ & $40(37.4 \%)$ & 0.042 \\
\hline $\operatorname{high}(\mathrm{n})$ & $63(60.6 \%)$ & $53(49.5 \%)$ & \\
\hline
\end{tabular}

Notes: variables are reported as "median (interquartile range)" and "value (percent of group)"; $p$ values represent the significance level of the test used to assess differences: Mann-Whitney (scale test variables); $\chi^{2}$ (nominal test variables).

Abbreviations: AAD/M - appendicular adipose density/mass; AT D/M/A/P - adipose tissue density/mass/area/percent; BMI - body mass index; FDR - fat distribution ratio; FMI - fat mass index; MetS - metabolic syndrome; NWO - normal weight obesity; POO - pre-obese obesity; RA - rheumatoid arthritis; TAD/M - trunk adipose density/mass; TPA - total physical activity; wb - whole body; W/HC - waist/hip circumference; WHR - waist-to-hip ratio. 


\subsection{RA-SPECIFIC VARIABLES}

Dividing the RA group into body composition phenotypes yielded significant differences between the respective subgroups regarding RA-specific variables (Table 2). Thus, overfat RA patients had a significantly higher toll of inflammation, disease activity, glucocorticoid treatment and sedentary behavior (median of $360 \mathrm{~min} /$ day compared to $240 \mathrm{~min} /$ day; $\mathrm{p}=0.045$ ). RA patients with the MetS had a lower prevalence of extra-articular manifestations $(15.4 \%$ compared to $33.3 \%$; $\mathrm{p}=$ $0.04)$ and a higher rate of DMARD $(95.4 \%$ compared to $80.9 \% ; \mathrm{p}=0.036)$ and $\mathrm{GC}(55.4 \%$ compared to $33.3 \% ; \mathrm{p}=0.042$ ) treatment. RA patients with POO had a higher rate of RF-positivity $(100 \%$ compared to $78.8 \% ; \mathrm{p}=0.005)$ and lower rate of DMARD treatment $(80.5 \%$ compared to $95.5 \% ; \mathrm{p}=0.045$ ).

Dividing the RA group into RA phenotypes also resulted in significantly different respective subgroups regarding RA-specific variables (Table 3). Generally, patients with inflammation, glucocorticoid treatment and higher disease activity class had higher medians of whole body adipose tissue indices and higher rates of dysmetabolic phenotypes. Patients with DAS28 $8_{\text {ESR }}$-MDA had a significantly higher median wbATP than patients with DAS28ESRremission $(65.7 \%$ compared to $55.2 \% ; \mathrm{p}=0.039)$.

Table 2

RA-specific variables and their differences in overfat patients

\begin{tabular}{|c|c|c|c|c|c|}
\hline & $\begin{array}{l}\text { all } \\
(n=107)\end{array}$ & $\begin{array}{l}\text { FMI2-overfat } \\
\text { no }(\mathrm{n}=81)\end{array}$ & yes $(n=26)$ & $\begin{array}{l}\text { wbATP-overfat } \\
\text { no }(\mathrm{n}=70)\end{array}$ & yes $(n=37)$ \\
\hline age (y) & $56(18)$ & $55(19)$ & $57(14)$ & $55(18)$ & $58(16)$ \\
\hline $\operatorname{ESR}(\mathrm{mm} / \mathrm{h})$ & $30(36)$ & $26(33)$ & $38(36)^{\S}$ & $25(31)$ & $42(43)^{\S}$ \\
\hline CRP (mg/L) & $6.8(18.8)$ & $4.4(14.9)$ & $17.2(24.1)^{\S}$ & $3.9(17.2)$ & $12.5(23.9)^{\S}$ \\
\hline inflammation (n) & $70(65.4 \%)$ & $47(58.1 \%)$ & $23(88.5 \%)^{\S}$ & $39(55.7 \%)$ & $31(83.8 \%)^{\S}$ \\
\hline RA duration (y) & $10(12)$ & $9(10)$ & $13(19)$ & $9(10)$ & $13(18)$ \\
\hline stiffness (min) & $30(80)$ & $30(80)$ & $30(80)$ & $30(120)$ & $30(45)$ \\
\hline joint deformity (n) & $77(71.9 \%)$ & $59(72.8 \%)$ & $18(69.2 \%)$ & $51(72.9 \%)$ & $26(70.3 \%)$ \\
\hline EAM (n) & $24(22.4 \%)$ & $19(23.5 \%)$ & $5(19.2 \%)$ & $18(25.7 \%)$ & $6(16.2 \%)$ \\
\hline $\mathrm{RN}(\mathrm{n})$ & $21(19.6 \%)$ & $16(19.8 \%)$ & $5(19.2 \%)$ & $15(21.4 \%)$ & $6(16.2 \%)$ \\
\hline $\mathrm{RF}+(\mathrm{n})$ & $93(86.9 \%)$ & $69(85.2 \%)$ & $24(92.3 \%)$ & $60(85.7 \%)$ & $33(89.2 \%)$ \\
\hline $\mathrm{ACPA}+(\mathrm{n})$ & $94(87.8 \%)$ & $71(87.7 \%)$ & $23(88.5 \%)$ & $60(85.7 \%)$ & $34(91.9 \%)$ \\
\hline DMARD* $^{*}(\mathrm{n})$ & $96(89.7 \%)$ & $70(86.4 \%)$ & $26(100 \%)^{\S}$ & $62(88.6 \%)$ & $34(91.9 \%)$ \\
\hline $\operatorname{biologics}^{\#}(\mathrm{n})$ & $35(32.7 \%)$ & $26(32.1 \%)$ & $9(34.6 \%)$ & $23(32.9 \%)$ & $12(32.4 \%)$ \\
\hline $\mathrm{GC}(\mathrm{n})$ & $49(45.8 \%)$ & $32(39.5 \%)$ & $17(65.4 \%)^{\S}$ & $28(40.0 \%)$ & $21(56.8 \%)^{\S}$ \\
\hline DAS $28_{\mathrm{ESR}}$ & $4.38(1.81)$ & $4.11(2.12)$ & $4.62(0.87)^{\S}$ & $4.01(2.21)$ & $4.64(1.05)^{\S}$ \\
\hline DAS $28_{\mathrm{CRP}}$ & $3.94(1.64)$ & $3.81(1.89)$ & $4.11(0.89)$ & $3.52(1.93)$ & $3.99(0.97)$ \\
\hline CDAI & $13.6(11.5)$ & $13.2(11.9)$ & $14.1(9.3)$ & $12.9(12.7)$ & $15.6(8.2)^{\S}$ \\
\hline HAQ & $0.63(0.75)$ & $0.63(0.88)$ & $0.88(0.55)$ & $0.56(0.75)$ & $0.86(0.56)^{\S}$ \\
\hline SENS & $22(28)$ & $21(22)$ & $39(43)$ & $21(19)$ & $36(39)^{\S}$ \\
\hline \multicolumn{6}{|c|}{ DAS2 $8_{E S R}$ disease activity $^{\xi}$} \\
\hline remission $(\mathrm{n})$ & $11(10.3 \%)$ & $11(13.6 \%)$ & $0(0 \%)$ & $11(15.7 \%)$ & $0(0 \%)$ \\
\hline low (n) & $13(12.1 \%)$ & $13(16.1 \%)$ & $0(0 \%)$ & $12(17.1 \%)$ & $1(2.7 \%)$ \\
\hline moderate (n) & $54(50.5 \%)$ & $34(41.9 \%)$ & $20(76.9 \%)$ & $28(40.0 \%)$ & $26(70.3 \%)$ \\
\hline $\operatorname{high}(\mathrm{n})$ & $29(27.1 \%)$ & $23(28.4 \%)$ & $6(23.1 \%)$ & $19(27.1 \%)$ & $10(27.1 \%)$ \\
\hline \multicolumn{6}{|c|}{ CDAI disease activity } \\
\hline remission (n) & $6(5.6 \%)$ & $6(7.4 \%)$ & $0(0 \%)$ & $6(8.6 \%)$ & $0(\%)$ \\
\hline low (n) & $25(23.4 \%)$ & $22(27.2 \%)$ & $3(11.5 \%)$ & $21(30.0 \%)$ & $4(10.8 \%)$ \\
\hline moderate (n) & $61(57 \%)$ & $42(51.9 \%)$ & $19(73.1 \%)$ & $34(48.6 \%)$ & $27(72.9 \%)$ \\
\hline high (n) & $15(14 \%)$ & $10(12.3 \%)$ & $5(19.2 \%)$ & $9(12.9 \%)$ & $6(16.2 \%)$ \\
\hline
\end{tabular}

Notes:

* methotrexate, leflunomide, sulfasalazine, hydroxychloroquine, azathioprine, cyclosporine;

\# infliximab, etanercept, adalimumab, golimumab, certolizumab, rituximab, abatacept, tocilizumab;

- variables are reported as "median (interquartile range)" and "value (percent of group)";

- the test used to assess differences were Mann-Whitney (scale test variables); $\chi^{2}$ (nominal test variables), with the following significance levels: $\S \mathrm{p}<0.05$; non-significant if unmarked.

Abbreviations: ACPA - anti-citrullinated protein antibodies; CDAI - clinical disease activity index; CRP - C reactive protein; DAS - disease activity score; DMARD - disease-modifying antirheumatic drugs; EAM - extra-articular manifestations; ESR - erythrocyte sedimentation rate; FMI - fat mass index; GC - glucocorticoids; HAQ - health assessment questionnaire; $\mathrm{n}$ - number (observed value); RA - rheumatoid arthritis; RF - rheumatoid factor; RN rheumatoid nodules; SENS - Simple Erosion Narrowing Score; wbATP - whole body adipose tissue percent; y - years. 
Table 3

Significant differences among RA subgroups

\begin{tabular}{|c|c|c|c|c|c|c|c|c|}
\hline & \multicolumn{2}{|l|}{ inflammation } & \multicolumn{2}{|c|}{ glucocorticoids } & \multicolumn{2}{|c|}{ CDAI activity } & \multicolumn{2}{|c|}{ joint deformity } \\
\hline & no $(n=37)$ & yes $(n=70)$ & no $(\mathrm{n}=58)$ & yes $(n=49)$ & $R(n=6)$ & $\mathrm{MDA}(\mathrm{n}=61)$ & no $(n=30)$ & yes $(n=77)$ \\
\hline wbATD $\left(\mathrm{g} / \mathrm{cm}^{2}\right)$ & $6.89(1.87)$ & $7.86(2.86)^{*}$ & $7.28(2.44)$ & $8.11(3.18)^{*}$ & $4.7(3.6)$ & $7.9(3.2)$ & $8.32(3.37)$ & $7.58(1.98)$ \\
\hline wbATM (kg) & 34.7 (11.9) & $40.7(19.5)^{*}$ & $37.9(16.9)$ & $43.5(19.8)^{*}$ & $18.8(25.7)$ & $40.8(17.8)^{*}$ & $46.3(19.4)$ & $39.2(14.8)^{*}$ \\
\hline wbATA $\left(m^{2}\right)$ & $0.52(0.05)$ & $0.53(0.07)$ & $0.52(0.07)$ & $0.53(0.05)$ & $0.44(0.16)$ & $0.53(0.05)$ & $0.55(0.05)$ & $0.51(0.06)^{*}$ \\
\hline wbATP (\%) & $57.8(10.2)$ & $65.2(14.5)^{*}$ & $59.5(11.7)$ & $63.3(12.9)^{*}$ & $48.1(19.8)$ & $65.1(12.6)^{*}$ & $62.5(13.6)$ & $63.4(14.3)$ \\
\hline $\operatorname{AAD}\left(\mathrm{g} / \mathrm{cm}^{2}\right)$ & $5.28(1.16)$ & $5.96(1.48)^{*}$ & $5.59(1.26)$ & $5.69(1.82)$ & $3.75(2.22)$ & $6.01(1.37)$ & $6.11(1.78)$ & $5.43(1.24)$ \\
\hline AAM $(\mathrm{kg})$ & $16.8(5.9)$ & $19.7(7.3)^{*}$ & $18.6(7.1)$ & $19.3(6.9)$ & $11.2(10.3)$ & $20.2(6.9)^{*}$ & $21.7(7.6)$ & $18.4(5.9)^{*}$ \\
\hline $\operatorname{ATD}_{\text {pekris }}\left(\mathrm{g} / \mathrm{cm}^{2}\right)$ & $10.06(3.71)$ & $11.67(3.87)^{*}$ & $10.7(4.2)$ & $11.5(4.2)^{*}$ & $6.71(4.75)$ & $11.77(3.45)$ & $12.4(4.6)$ & $10.8(3.5)^{*}$ \\
\hline $\mathrm{ATM}_{\mathrm{Pek} \text { kis }}(\mathrm{kg})$ & $6.68(2.86)$ & $8.27(3.87)^{*}$ & $7.1(3.5)$ & $8.3(4.7)^{*}$ & $3.2(4.8)$ & $8.1(3.8)^{*}$ & $8.9(5.4)$ & $7.4(3.1)^{*}$ \\
\hline FMI1 (\%) & $53.7(10.5)$ & $59.6(14.3)^{*}$ & $55.2(12.3)$ & $59.1(13.5)^{*}$ & $43.3(22.4)$ & $58.8(12.9)$ & $57.7(13.7)$ & $58.4(14.2)$ \\
\hline FMI2 $\left(\mathrm{kg} / \mathrm{m}^{2}\right)$ & $14.08(4.63)$ & $16.34(7.29)^{*}$ & $15.4(5.5)$ & $15.5(8.2)$ & $8.2(8.4)$ & $16.3(6.3)^{*}$ & $17.2(6.9)$ & $15.3(5.4)^{*}$ \\
\hline FDR2 (\%) & $40.2(10.6)$ & $41.1(11.8)$ & $37.4(11.2)$ & $42.7(10.8)^{*}$ & $31.8(11.9)$ & $41.9(9.9)$ & $40.1(12.1)$ & $40.9(10.3)$ \\
\hline FMI2-overfat (n) & $3(8.1 \%)$ & $23(32.9 \%)^{*}$ & $9(15.5 \%)$ & $17(34.7 \%)^{*}$ & $0(0 \%)$ & $18(29.5 \%)$ & $9(30.0 \%)$ & $17(22.1 \%)$ \\
\hline wbATP-overfat (n) & $6(16.2 \%)$ & $31(44.3 \%)^{*}$ & $16(27.6 \%)$ & $22(44.9 \%)^{*}$ & $0(0 \%)$ & $27(44.3 \%)^{*}$ & $12(40.0 \%)$ & $26(33.8 \%)$ \\
\hline POO (n) & $17(45.9 \%)$ & $30(42.9 \%)$ & $28(48.3 \%)$ & $18(36.7 \%)$ & $1(16.7 \%)$ & $24(39.3 \%)$ & $12(40.0 \%)$ & $34(44.2 \%)$ \\
\hline MetS (n) & $24(64.8 \%)$ & $40(57.1 \%)$ & $28(48.3 \%)$ & $34(69.4 \%)^{*}$ & $1(16.7 \%)$ & $37(60.7 \%)^{*}$ & $14(46.7 \%)$ & $50(64.9 \%)^{*}$ \\
\hline
\end{tabular}

Note: variables are reported as "median (interquartile range)" and their differences are assessed with Mann-Whitney and Kruskal-Wallis tests which are significant $(\mathrm{p}<0.05)$ if marked with *

Abbreviations: AAD/M - appendicular adipose density/mass; ATD/M/A/P - adipose tissue density/mass/area/percent; CDAI - clinical disease activity index; FDR - fat distribution ratio; FMI - fat mass index; MetS - metabolic syndrome; $\mathrm{n}$ - number; POO - pre-obese obesity; RA - rheumatoid arthritis; wb - whole body.

When assessing these differences, replacing absolute values of DXA adipose tissue with those obtained by dividing them either with $\mathrm{WC}$ or $\mathrm{HC}$ resulted in an increase of statistical significance of the above differences and the appearance of others with the same pattern (data not shown).

\subsection{PREDICTION OF WHOLE BODY ADIPOSE TISSUE}

Glucocorticoid treatment and RA variables such as inflammation, disease duration and severity (HAQ, SENS, activity indices) correlated with whole body adipose tissue in RA patients and proved to be significant predictors and risk factors for high adiposity content and overfat phenotypes (Table 4, Figure 1). RA patients with joint deformity behaved differently. On one hand they had significantly lower whole body adiposity and on the other hand, joint deformity classification was negatively correlated with fat content (significant) and overfat phenotype (non-significant).

\section{DISCUSSION}

\subsection{RA VERSUS CONTROLS}

Our data reveal several particularities of whole body adiposity of RA women. Even though controls had a higher median BMI and a higher rate of BMI-defined obesity, anthropometrical measurements of trunk adiposity (WC, HC, WHR) and DXA measures of whole body, appendicular and trunk adiposity did not differ significantly, indicating the tendency of central redistribution of adiposity in RA. In other words, even though they weighed less, RA women had the same amount of body fat compared to controls. Similarly, RA women had a significantly higher rate of BMI-defined normal weight but at the same time they also had a significantly higher rate of NWO, illustrating an altered body composition for the same BMI category. Most DXA studies have found higher whole body 
and regional adipose tissue indices in RA women compared to controls [1,3,4], although others reported no such significant differences [29, 30]. This discrepancy is probably explained by the fact that most of these studies, including our own, are done on relatively small samples from culturally different populations, which may cause a significant variation of body composition in controls.

Table 4

Significant RA predictors of lean tissue scale indices

\begin{tabular}{|c|c|c|c|c|c|c|c|}
\hline & dependent & independent & $r$ & $R^{2}$ & $B$ & $S E$ & B $95 \% C I$ \\
\hline \multirow{24}{*}{ 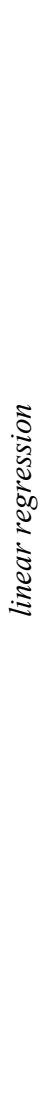 } & AAD & $\ln (\mathrm{ESR})$ & 0.27 & 0.06 & 0.37 & 0.18 & $0.02-0.72$ \\
\hline & AAM & $\ln (\mathrm{ESR})$ & 0.26 & 0.06 & 1.53 & 0.72 & $0.01-2.97$ \\
\hline & wbATP & $\ln (\mathrm{ESR})$ & 0.23 & 0.05 & 2.52 & 1.38 & $0.23-5.27$ \\
\hline & wbATD & $\ln (\sqrt{\mathrm{CRP}})$ & 0.23 & 0.05 & 0.56 & 0.29 & $0.02-1.15$ \\
\hline & wbATM & $\ln (\sqrt{\mathrm{CRP}})$ & 0.24 & 0.06 & 3.56 & 1.66 & $0.25-6.87$ \\
\hline & wbATP & $\ln (\sqrt{\mathrm{CRP}})$ & 0.25 & 0.05 & 0.03 & 0.01 & $0.01-0.09$ \\
\hline & $\mathrm{ATM}_{\text {pelvis }}$ & $\ln (\sqrt{\mathrm{CRP}})$ & 0.25 & 0.05 & 0.95 & 0.51 & $0.07-1.96$ \\
\hline & AAD & $\ln \left(\sqrt{\mathrm{CRP}^{3}}\right)$ & 0.24 & 0.06 & 0.38 & 0.19 & $0.01-0.75$ \\
\hline & AAM & $\ln (\sqrt{\mathrm{CRP}})$ & 0.28 & 0.07 & 1.73 & 0.76 & $0.22-3.24$ \\
\hline & FMI2 & $\ln (\sqrt{\mathrm{CRP}})$ & 0.24 & 0.07 & 1.42 & 0.63 & $0.17-2.67$ \\
\hline & wbATP & RA duration & 0.26 & 0.07 & 0.24 & 0.11 & $0.03-0.44$ \\
\hline & wbATP & $\sin (H A Q)$ & 0.26 & 0.07 & 7.81 & 3.26 & $1.31-9.29$ \\
\hline & wbATP & SENS & 0.29 & 0.08 & 0.14 & 0.05 & $0.04-0.25$ \\
\hline & FMI1 & SENS & 0.29 & 0.08 & 0.13 & 0.05 & $0.03-0.23$ \\
\hline & wbATD & DAS28 $8_{\text {ESR }}$ class & - & 0.06 & 1.17 & 0.52 & $0.13-2.21$ \\
\hline & wbATM & DAS28 $8_{\text {ESR }}$ class & - & 0.06 & 7.68 & 3.56 & $0.61-1.47$ \\
\hline & AAD & DAS28 $8_{\text {ESR }}$ class & - & 0.06 & 0.68 & 0.33 & $0.02-1.34$ \\
\hline & AAM & joint deformity & - & 0.06 & -2.82 & 1.26 & $-5.3--0.3$ \\
\hline & AAM & DAS28 $8_{\text {ESR }}$ class & - & 0.07 & 3.16 & 1.35 & $0.47-5.85$ \\
\hline & AAM & CDAI class & - & 0.05 & 2.54 & 1.25 & $0.05-5.03$ \\
\hline & $\mathrm{ATD}_{\text {pelvis }}$ & DAS28 $8_{\mathrm{ESR}}$ class & - & 0.06 & 1.74 & 0.83 & $0.09-3.39$ \\
\hline & $\mathrm{ATM}_{\text {pelvis }}$ & glucocorticoids & - & 0.06 & 1.61 & 0.75 & $0.11-3.11$ \\
\hline & $\mathrm{ATM}_{\text {pelvis }}$ & DAS28 $8_{\text {ESR }}$ class & - & 0.05 & 1.83 & 0.91 & $0.03-3.63$ \\
\hline & dependent & independent & $O R$ & $R^{2}$ & $B$ & $S E$ & OR 95\% CI \\
\hline \multirow{9}{*}{ 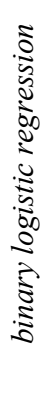 } & FMI2-overfat & CRP & 1.87 & 0.08 & 0.95 & 0.48 & $1.19-3.21$ \\
\hline & FMI2-overfat & inflammation & 5.65 & 0.12 & 1.73 & 0.79 & $1.39-6.88$ \\
\hline & FMI2-overfat & glucocorticoids & 3.04 & 0.08 & 1.11 & 0.57 & $1.61-9.26$ \\
\hline & wbATP-overfat & ESR & 1.42 & 0.09 & 0.45 & 0.30 & $1.11-3.07$ \\
\hline & wbATP-overfat & DAS28 $8_{\mathrm{ESR}}$ & 1.55 & 0.08 & 0.44 & 0.22 & $1.31-2.39$ \\
\hline & wbATP-overfat & HAQ & 3.98 & 0.07 & 0.89 & 0.56 & $1.51-6.05$ \\
\hline & wbATP-overfat & SENS & 1.04 & 0.17 & 0.03 & 0.01 & $1.00-1.06$ \\
\hline & wbATP-overfat & inflammation & 4.32 & 0.12 & 1.46 & 0.61 & $1.29-7.39$ \\
\hline & wbATP-overfat & DAS28 $8_{\mathrm{ESR}}$ class & 4.77 & 0.17 & 2.47 & 1.06 & $1.46-6.62$ \\
\hline
\end{tabular}

Notes: the $r$ column reports two-tailed partial correlations; the other columns report standard multivariate linear regression models (for scale dependents) and binary logistic regression models (for nominal dependents); both correlations and regression models are significant $(\mathrm{p}<0.05)$ and are adjusted for age, BMI, physical activity, glucocorticoids and smoking status (confounders added in the models); nominal variables are coded " 0 " for "no" or "remission and LDA" and "1" for "yes" or "MDA and HDA" respectively.

Abbreviations: AAD/M - appendicular adipose density/mass; wbATD/M/P - whole body adipose tissue density/ mass/percent; BMI - body mass index; CDAI - clinical disease activity index; CI - confidence interval; CRP C-reactive protein; ESR - erythrocyte sedimentation rate; FMI - fat mass index; HAQ - health assessment questionnaire; L/M/HAD - low/moderate/high disease activity; OR - odds ratio; RA - rheumatoid arthritis; SE standard error; SENS - Simple Erosion Narrowing Score. 

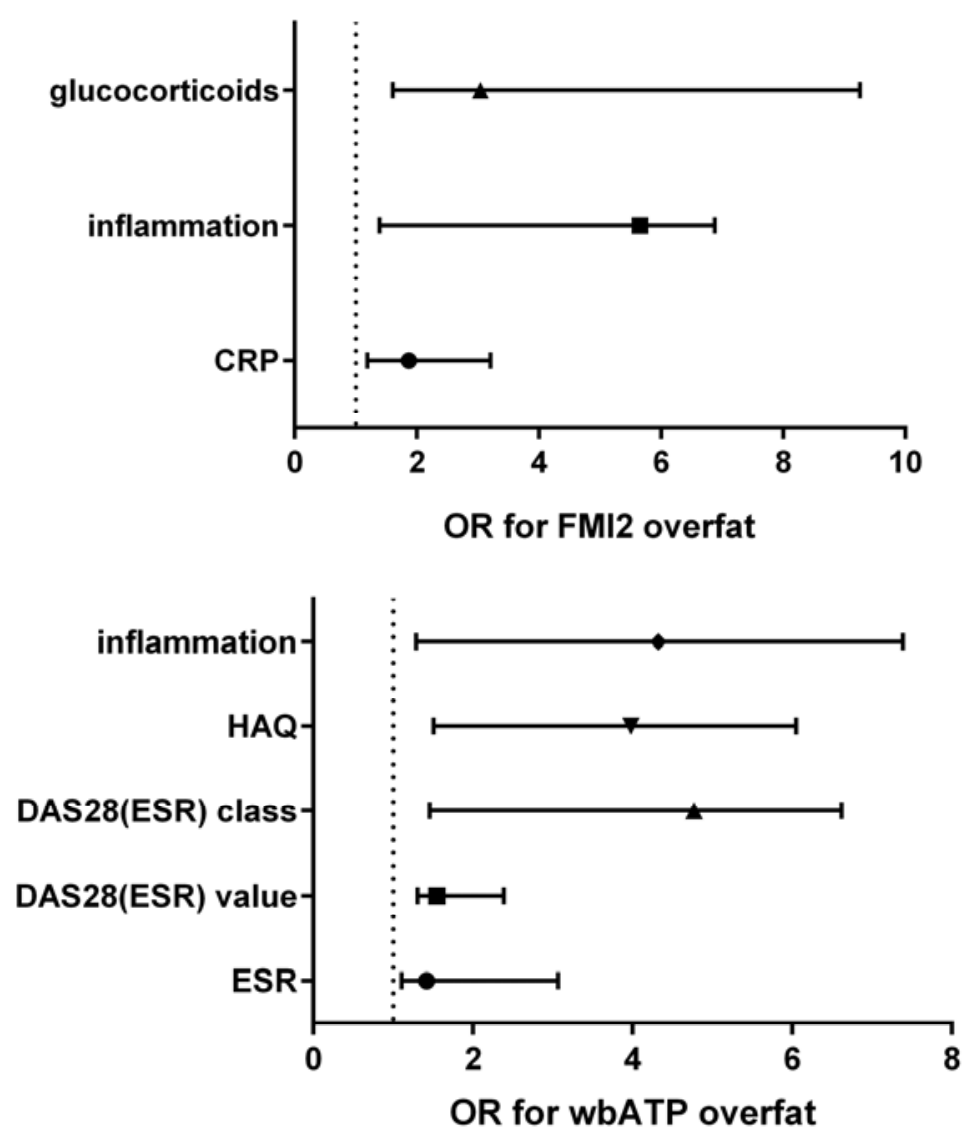

Figure 1. Significant risk factors for overfat status in RA patients (defined by two different DXA measures). Abbreviations: CRP - C-reactive protein; DAS - disease activity score; ESR - erythrocyte sedimentation rate; FMI - fat mass index; HAQ - health assessment questionnaire; OR - odds ratio; RA - rheumatoid arthritis; wbATP - whole body adipose tissue percent.

\subsection{RA VARIABLES}

Inflammation seems to be an important driving factor of whole body fat in RA. Compared to RA women with normal ESR and CRP, RA women with inflammation had a higher prevalence of overfat status and higher amounts of whole body and regional adiposity. Additionally, inflammation itself and inflammation markers were significant predictors and risk factors for overfat status and high whole body and regional fat content $[7,14$, 31]. Conversely, overfat RA women had a higher prevalence of inflammation [1]. These quantitative and qualitative observations of a bidirectional link between inflammation and adipose tissue are in accordance with the modern pathogenic concepts of obesity as an inflammatory disorder [32, 33]. Since inflammation is inherently linked to disease activity, one would expect a significant influence of disease activity on the fat content. Indeed, in the same bidirectional manner, our overfat RA women had higher values and classes of disease activity indices, while a higher activity score or class was associated and predicted a higher adipose tissue value or state (overfat) $[3,4,31]$. The influence of inflammation and disease activity on whole body adiposity would be better assessed if one would be able to record the time duration of high inflamematory markers and high disease activity. Longstanding inflammation and high disease activity typically leads to RA joint deformity. The severity of this evolution seems to be accompanied by changes in adipose body composition. In our study, overfat RA patients had a longer disease duration and a more severe disease (HAQ, SENS, joint deformity), which were also significant predictors of adipose tissue indices and states (overfat). The significance level of these variables for predicting adiposity varies in the literature $[3,4,7,14]$, but all the studies show a positive statistical relationship with whole body and trunk adiposity. Uniquely, our RA patients with joint deformity behaved differently: they had lower whole body and regional adipose tissue indices than RA patients without joint deformity, a pattern which was also apparent from the prediction study (although statistically significant only for appendicular fat). Upon inspecting this subgroup, we found that these patients were 
significantly older; they had a longer disease duration and lower body mass and BMI. Either these patients have geriatric cachexia or that is the natural course of RA-induced body composition alteration, since joint deformity is a marker of longstanding or uncontrolled disease and since it can lead to sedentary behavior, chronic pain, disability, nutrition disturbances and depression.

Treatment regimes are also associated with modification of adipose tissue quantity and distribution in RA, either directly (glucocorticoids) or indirectly by suppressing inflammation and disease activity. The reports on glucocorticoids are contradicting: some authors find as expected a positive link with whole body and/or trunk adiposity [4, 14], while others report a non-significant influence [3, 7, 29, 30]. In our sample glucocorticoid treatment made significant influences: overfat RA women had a higher frequency of glucocorticoid treatment which in turn predicted overfat status, while patients on glucocorticoids had higher whole body and regional adiposity. Since low doses of glucocorticoids can determine osteoporosis and other side effects, the gain and redistribution of fat seems a highly likely event in glucocorticoid-treated RA women. There are reports of beneficial effect of DMARD treatment on adiposity [4] and a detrimental effect of autoimmunity seropositivity $[3,4]$, findings which were not confirmed by our data.

\subsection{STUDY LIMITATIONS}

The design of the study did not allow followup of patients and dynamic observations of whole body adipose tissue. Data regarding diet and insulin resistance were not included in the study design. For a more thorough comparison between RA patients and controls regarding adipose tissue indices, a BMI-match may have increased the objectivity of the observation. Since the number of comparisons between variables was very high, there is an increased risk of a type I error (alpha).

\section{CONCLUSION}

Women with RA have a higher prevalence of normal weight obesity and exercise less compared to controls. Overfat status in RA women is associated with a significantly higher toll of inflammation, disease activity, glucocorticoid treatment and sedentary behavior. Glucocorticoids, disease activity and severity are associated and predict overfat status and whole body and regional adipose tissue indices. RA women with joint deformity and longstanding disease tend to have lower body mass and whole body adiposity. Clinicians may need to assess body composition and physical activity in RA patients in order to fully manage cardiovascular outcomes and quality of life.

Conflict of interest. No conflicts of interest relevant to this article are declared.

Acknowledgements. The study was supported by the European Social Fund, contract number POSDRU/159/1.5/ S/137390. The author would like to thank Irina Ban M.D. for excellent technical expertise in translating the physical activity questionnaire.

Obiective. Poliartrita reumatoidă (PR) ar putea influența nu numai adipozitatea abdominală, ci şi pe cea totală, întrucât ea se asociază cu inflamație cronică şi dizabilitate. Studiul îşi propune să evalueze țesutul adipos corporal total PR şi să cuantifice influența variabilelor specifice bolii asupra adipozității totale.

Metode. Studiul a inclus femei cu PR în postmenopauză şi subiecți normali de sex feminin şi vârste similare. Fiecare participant a efectuat într-o singură zi examinare clinică, teste de laborator, estimarea compoziției de masă corporală cu absorbțiometria duală cu raze X (DXA) şi evaluarea activității fizice folosind un chestionar auto-administrat.

Rezultate. In total, studiul a inclus 107 de paciente cu PR şi 104 subiecți normali. In comparație cu subiecții normali, pacientele cu PR prezentau nivele mai scăzute de activitate fizică şi o prevalență semnificativ mai mare a obezității normoponderale. Pacientele cu exces de țesut adipos (,overfat”) prezentau un nivel mai ridicat al inflamației, activității bolii, frecvenței terapiei cu glucocorticoizi şi frecvenței comportamentului sedentar. Pacientele cu inflamație, cele tratate cu glucocorticoizi şi cele cu activitate înaltă a bolii prezentau indici ai țesutului adipos total şi abdominal semnificativ mai mari, precum şi o prevalență 
mai ridicată a statutului de „overfat”. Glucocorticoizii, inflamația, durata şi severitatea bolii s-au corelat cu adipozitatea totală şi au fost factori predictivi semnificativi ai conținutului de țesut adipos şi al fenotipului ,,overfat”.

Concluzii. Durata şi severitatea PR se asociază cu adipozitate totală şi regională semnificativ mai mare. Tratamentul cu glucocorticoizi, chiar şi în doză mică, contribuie la adiția şi redistribuirea adipozității. Practicienii ar putea evalua compoziția de masă folosind DXA la pacientele cu PR în scopul unui management cardiovascular complet al acestor paciente.

Correspondence to: Claudiu Popescu, "Sfânta Maria" Clinical Hospital,

37-39 Ion Mihalache Boulevard, Bucharest, district 1, postal code 011192

Tel. +040729852359

E-mail: dr.reumatologie@gmail.com

\section{REFERENCES}

1. GILES JT, LING SM, FERRUCCI L, BARTLETT SJ, ANDERSEN RE, TOWNS M et al. Abnormal body composition phenotypes in older rheumatoid arthritis patients: association with disease characteristics and pharmacotherapies. Arthritis Rheum 2008; 59: 807-815.

2. BOOK C, KARLSSON MK, NILSSON JA, AKESSON K, JACOBSSON LT. Changes in body composition after 2 years with rheumatoid arthritis. Scand J Rheumatol 2011; 40: 95-100.

3. BOOK C, KARLSSON MK, AKESSON K, JACOBSSON LT. Early rheumatoid arthritis and body composition. Rheumatology (Oxford) 2009; 48: 1128-1132.

4. DAO HH, DO QT, SAKAMOTO J. Abnormal body composition phenotypes in Vietnamese women with early rheumatoid arthritis. Rheumatology (Oxford) 2011; 50: 1250-1258.

5. STAVROPOULOS-KALINOGLOU A, METSIOS GS, KOUTEDAKIS Y, KITAS GD. Obesity in rheumatoid arthritis. Rheumatology (Oxford) 2011; 50: 450-462.

6. STAVROPOULOS-KALINOGLOU A, METSIOS GS, KOUTEDAKIS Y, NEVILL AM, DOUGLAS KM, JAMURTAS A $e t$ al. Redefining overweight and obesity in rheumatoid arthritis patients. Ann Rheum Dis 2007; 66: 1316-1321.

7. ELKAN AC, ENGVALL IL, CEDERHOLM T, HAFSTROM I. Rheumatoid cachexia, central obesity and malnutrition in patients with low-active rheumatoid arthritis: feasibility of anthropometry, Mini Nutritional Assessment and body composition techniques. Eur J Nutr 2009; 48: 315-322.

8. BILECIK NA, TUNA S, SAMANCI N, BALCI N, AKBAS H. Prevalence of metabolic syndrome in women with rheumatoid arthritis and effective factors. Int J ClinExp Med 2014;7: 2258-2265.

9. DA C, V, BRENOL CV, BRENOL JC, FUCHS SC, ARLINDO EM, MELO IM et al. Metabolic syndrome prevalence is increased in rheumatoid arthritis patients and is associated with disease activity. Scand J Rheumatol 2012; 41: 186-191.

10. OGDIE A, YU Y, HAYNES K, LOVE TJ, MALIHA S, JIANG Y et al. Risk of major cardiovascular events in patients with psoriatic arthritis, psoriasis and rheumatoid arthritis: a population-based cohort study. Ann Rheum Dis 2014.

11. CERHAN JR, SAAG KG, CRISWELL LA, MERLINO LA, MIKULS TR. Blood transfusion, alcohol use, and anthropometric risk factors for rheumatoid arthritis in older women. J Rheumatol 2002; 29: 246-254.

12. KONIJN NP, VAN TUYL LH, BULTINK IE, LEMS WF, EARTHMAN CP, VAN BOKHORST-DE VAN DER SCHUEREN MA. Making the invisible visible: bioelectrical impedance analysis demonstrates unfavourable body composition in rheumatoid arthritis patients in clinical practice. Scand J Rheumatol 2014; 43: 273-278.

13. LOMBARD LA, DU PLESSIS LM, VISSER J. Body composition of rheumatoid arthritis patients in the City of Cape Town, South Africa. Clin Rheumatol 2014; 33: 467-476.

14. ENGVALL IL, BRISMAR K, HAFSTROM I, TENGSTRAND B. Treatment with low-dose prednisolone is associated with altered body composition but no difference in bone mineral density in rheumatoid arthritis patients: a controlled cross-sectional study. Scand J Rheumatol 2011; 40: 161-168.

15. PODENPHANT J, GOTFREDSEN A, ENGELHART M, ANDERSEN V, HEITMANN BL, KONDRUP J. Comparison of body composition by dual energy X-ray absorptiometry to other estimates of body composition during weight loss in obese patients with rheumatoid arthritis. Scand J Clin Lab Invest 1996; 56: 615-625.

16. ALETAHA D, NEOGI T, SILMAN AJ, FUNOVITS J, FELSON DT, BINGHAM CO, III et al. 2010 Rheumatoid arthritis classification criteria: an American College of Rheumatology/European League Against Rheumatism collaborative initiative. Arthritis Rheum 2010; 62: 2569-2581.

17. PINCUS T, YAZICI Y, BERGMAN M. Development of a multi-dimensional health assessment questionnaire (MDHAQ) for the infrastructure of standard clinical care. Clin Exp Rheumatol 2005; 23: S19-S28.

18. VAN DER HD, DANKERT T, NIEMAN F, RAU R, BOERS M. Reliability and sensitivity to change of a simplification of the Sharp/van der Heijde radiological assessment in rheumatoid arthritis. Rheumatology (Oxford) 1999; 38: 941-947.

19. PREVOO ML, VAN 'T HOF MA, KUPER HH, VAN LEEUWEN MA, VAN DE PUTTE LB, VAN RIEL PL. Modified disease activity scores that include twenty-eight-joint counts. Development and validation in a prospective longitudinal study of patients with rheumatoid arthritis. Arthritis Rheum 1995; 38: 44-48. 
20. ALETAHA D, NELL VP, STAMM T, UFFMANN M, PFLUGBEIL S, MACHOLD K et al. Acute phase reactants add little to composite disease activity indices for rheumatoid arthritis: validation of a clinical activity score. Arthritis Res Ther 2005; 7: R796-R806.

21. LOHMAN M, TALLROTH K, KETTUNEN JA, MARTTINEN MT. Reproducibility of dual-energy x-ray absorptiometry total and regional body composition measurements using different scanning positions and definitions of regions. Metabolism 2009; 58: $1663-1668$.

22. DE LA, MARTINOLI R, VAIA F, DI RL. Normal weight obese (NWO) women: an evaluation of a candidate new syndrome. NutrMetabCardiovasc Dis 2006; 16: 513-523.

23. ALBERTI KG, ZIMMET P, SHAW J.Metabolic syndrome--a new world-wide definition. A Consensus Statement from the International Diabetes Federation. Diabet Med 2006; 23: 469-480.

24. GALLAGHER D, HEYMSFIELD SB, HEO M, JEBB SA, MURGATROYD PR, SAKAMOTO Y. Healthy percentage body fat ranges: an approach for developing guidelines based on body mass index. Am J ClinNutr 2000; 72: 694-701.

25. SCHUTZ Y, KYLE UU, PICHARD C. Fat-free mass index and fat mass index percentiles in Caucasians aged 18-98 y. Int J ObesRelatMetabDisord 2002; 26: 953-960.

26. ABERNATHY RP, BLACK DR. Healthy body weights: an alternative perspective. Am J ClinNutr 1996; 63: 448S-451S.

27. ARMSTRONG T, BULL F. Development of the World Health Organization Global Physical Activity Questionnaire (GPAQ). Journal of Public Health 2006; 14: 66-70.

28. BULL FC, MASLIN TS, ARMSTRONG T. Global physical activity questionnaire (GPAQ): nine country reliability and validity study. J Phys Act Health 2009; 6: 790-804.

29. WESTHOVENS R, NIJS J, TAELMAN V, DEQUEKER J. Body composition in rheumatoid arthritis. Br J Rheumatol 1997; 36: $444-448$

30. AKAR S, SARI I, COMLEKCI A, BIRLIK M, ONEN F, GOKTAY Y et al. Body composition in patients with rheumatoid arthritis is not different than healthy subjects. European Journal of Rheumatology 2014; 1: 106-110.

31. KATZ PP, YAZDANY J, TRUPIN L, SCHMAJUK G, MARGARETTEN M, BARTON J, et al. Sex differences in assessment of obesity in rheumatoid arthritis. Arthritis Care Res (Hoboken) 2013; 65: 62-70.

32. GREGOR MF, HOTAMISLIGIL GS. Inflammatory mechanisms in obesity. Annu Rev Immunol 2011; 29: 415-445.

33. LUMENG CN, SALTIEL AR.Inflammatory links between obesity and metabolic disease. J Clin Invest 2011; 121: 2111-2117.

Received May 9, 2015 